\title{
PERSPECTIVE
}

\section{A Research Agenda for Advancing Non-pharmacological Management of Chronic Musculoskeletal Pain: Findings from a VHA State-of-the-art Conference}

\author{
William C. Becker, MD ${ }^{1,2}$, Lynn L. DeBar, PhD ${ }^{3}$, Alicia A. Heapy, PhD ${ }^{1,2}$, Diana Higgins, PhD ${ }^{4,5}$,

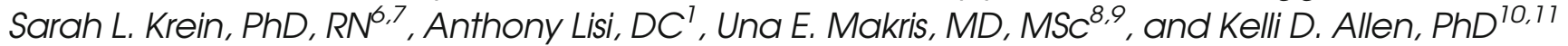 \\ ${ }^{1}$ Pain Research, Informatics, Multimorbidities \& Education Center of Innovation, VA Connecticut Healthcare System, West Haven, CT, USA; ${ }^{2}$ Yale \\ School of Medicine, New Haven, CT, USA; ${ }^{3}$ Kaiser Permanente Washington Health Research Institute, Seattle, WA, USA; ${ }^{4}$ VA Boston Healthcare \\ System, Boston, MA, USA; ${ }^{5}$ Boston University School of Medicine, Boston, MA, USA; ${ }^{6}$ Center for Clinical Management Research, VA Ann Arbor \\ Healthcare System, Ann Arbor, MI, USA; ${ }^{7}$ Department of Internal Medicine, University of Michigan School of Medicine, Ann Arbor, MI, USA; ${ }^{8}$ VA North \\ Texas Health Care System, Dallas, TX, USA; ${ }^{9}$ Department of Internal Medicine, UT Southwestern Medical Center, Dallas, TX, USA; ${ }^{10}$ Center for Health \\ Services Research in Primary Care, Durham VA Healthcare System, Durham, NC, USA; " Department of Medicine \& Thurston Arthritis Research \\ Center, University of North Carolina, Chapel Hill, NC, USA.
}

Chronic pain is widely prevalent among Veterans and can have serious negative consequences for functional status and quality of life among other domains. The Veterans Health Administration (VHA) convened a state-of-the-art (SOTA) conference to develop research priorities for advancing the science and clinical practice of nonpharmacological management of chronic musculoskeletal pain. In this perspective article, we present the methods and consensus recommendations for research priorities emanating from the SOTA. In the months leading up to the SOTA, a core group of researchers defined four areas of focus: psychological/behavioral therapies; exercise/movement therapies; manual therapies; and models for delivering multi-modal pain care and divided into workgroups. Each workgroup, in their respective areas of focus, identified seminal studies capturing the state of the evidence. Herein, we present consensus recommendations ranging from efficacy to effectiveness to implementation/dissemination research depending on the state of the evidence as assessed by participants, including commentary on common elements across workgroups and future areas of innovation in study design, measurement, and outcome ascertainment.

KEY WORDS: chronic pain management; non-pharmacologic treatments; clinical research.

J Gen Intern Med 33(Suppl 1):S11-S15

DOI: $10.1007 / \mathrm{s} 11606-018-4345-6$

(c) Society of General Internal Medicine 2018

\section{INTRODUCTION}

Chronic pain is widely prevalent among Veterans and can have serious negative consequences for an individual's functional status and quality of life. ${ }^{1}$ Furthermore, a primary treatment modality of the last two decades-long-term opioid therapy - has limited effectiveness and potential for serious

Prior presentations A summary of the SOTA was presented as a VA HSR\&D Spotlight on Pain Management Webinar in March, 2017.

Published online April 9, 2018 harm. $^{2}$ Accordingly, the Centers for Disease Control and Prevention and other stakeholders have promulgated recommendations seeking to shift healthcare systems away from opioid-centric chronic pain management and towards multimodal treatment paradigms where evidenced-based nonpharmacologic treatments are promoted. ${ }^{3,4}$ To this end, the Veterans Health Administration (VHA) convened a state-ofthe-art (SOTA) conference to develop research priorities for advancing the science and clinical practice of nonpharmacological management of chronic musculoskeletal pain. Here we present the methods and consensus recommendations for research priorities emanating from the SOTA.

\section{METHODS}

In the 6-month period leading up to the SOTA, co-chairs recruited a core group of researchers comprising the SOTA planning committee. Through a series of teleconferences, the committee reached consensus on four areas of focus-psychological/behavioral therapies; exercise/ movement therapies; manual therapies; and models for delivering multi-modal pain care, divided into four workgroups, and refined key questions to address SOTA objectives.

Each workgroup convened a series of teleconferences to further refine key questions, identify subject matter experts (an expanded group of clinicians, researchers, and policymakers) to participate in the SOTA, and select pre-conference readings the larger group of SOTA participants were assigned. The entire planning committee met periodically to discuss the progress of each workgroup. Pre-conference readings were chosen by workgroup consensus, prioritizing recency of publication; high-impact, high-quality individual studies or systematic reviews (if available); and that, taken together, each workgroup's core set of 6-10 recommended readings would promote a shared understanding of the state of the evidence among SOTA participants. Formal literature searches were not 
performed but each workgroup consisted of expert researchers in that area. SOTA attendees were assigned to one of the four workgroups and sent the pre-conference readings along with a list of key questions to help prepare for their workgroup discussion.

At the SOTA, participants were first briefed on the overall objectives followed by workgroup leaders facilitating breakout group discussion to address key questions and to reach consensus on research, clinical, and policy priorities. Next, the workgroups developed summaries of their deliberations. On the second day, these summaries were presented to all SOTA participants, providing opportunity for clarifying questions and discussion. Finally, there was an expert panel response to workgroup summaries followed by further full group discussion. Below we present the findings of each workgroup with respect to research gaps and priorities as well as our post hoc assessment of cross-cutting research priorities.

\section{PSYCHOLOGICAL/BEHAVIORAL THERAPIES}

In reviewing the psychological and behavioral treatments commonly provided in the VA, the workgroup focused on distinguishing between those treatments with strong efficacy/ effectiveness evidence where implementation research and dissemination are likely to be more beneficial and should be prioritized over additional efficacy/effectiveness research. These treatments include cognitive behavioral therapy, ${ }^{5-7}$ acceptance and commitment therapy, ${ }^{8,9}$ and mindfulness-based stress reduction. ${ }^{10}$ Other treatments were deemed to have sufficient evidence to support their clinical use, but additional efficacy/effectiveness research is warranted to address remaining gaps and weaknesses in the literature prior to concentrating solely on implementation research. These treatments include meditation, biofeedback, hypnosis, and relaxation therapies. Implementation trials should examine strategies to address major barriers to uptake of evidence-based psychological treatments such as low rates of patient engagement (often due to limited understanding of how or if psychological treatments can improve pain); high degree of patient burden (e.g., time, travel) associated with these treatments; and perceived stigma associated with psychological interventions. ${ }^{10,11}$ Additionally, clinicians may lack knowledge regarding psychological interventions, reducing their comfort in referring to such programs. ${ }^{11}$ Identified strategies with potential promise for enhancing intervention uptake in future implementation work include the use of motivational interviewing, treatment tailoring, improved patient messaging, and use of technology to provide in-home treatments. ${ }^{12,13}$

The workgroup identified several additional knowledge gaps and areas for future research. Given that Veterans with chronic pain frequently present with comorbid mental health conditions that may interfere with treatment response, ${ }^{14}$ integrated treatments (e.g., for pain and depression) may be less burdensome and more effective for patients with comorbid conditions. Because alternate treatment modalities (telehealth vs. in-person, group vs. individual, and brief vs. standard) may make interventions more accessible to patients, research on the impact of treatment delivery mode is a priority. Finally, treatments often consist of a single modality; however, there may be additive benefit to treatment approaches that combine modalities (e.g., psychological and exercise) relative to single modality approaches.

\section{EXERCISE/MOVEMENT-BASED THERAPIES}

This workgroup reviewed the evidence for a broad array of program types, with an emphasis on interventions that could be delivered or facilitated by providers (i.e., physical therapists) and other individuals within the VHA. The workgroup found there is sufficient evidence to recommend deployment of three types of exercise within the VHA: Tai Chi, ${ }^{15-17}$ yoga, ${ }^{18-20}$ and exercise therapies including coordination/ stabilization, strengthening/resistance, and aerobic/ cardiorespiratory activities. ${ }^{17},{ }^{21-25}$ The workgroup concluded that further study is needed regarding the effectiveness of aquatic exercise for low back pain and fibromyalgia, although evidence may be sufficient to recommend it for osteoarthritis pain. ${ }^{26}$ There is also some evidence for balance and proprioception exercises for patients with osteoarthritis.

The workgroup identified evidence gaps related to implementation of evidence-based exercise/movement-based therapies that should be considered in the design of future studies including lack of standards for training and background of personnel delivering programs, lack of available standardized protocols to facilitate fidelity for some types of interventions, and sparse data comparing the effectiveness of delivery modalities (e.g., group, individual, telehealth). Several crosscutting issues in need of further investigation across numerous types of exercise were identified that closely mirrored other groups' findings; as such, they will be discussed in the "crosscutting research" section below. Lastly, the workgroup recognized that access to evidence-based exercise/movement therapies is often limited; thus, future research should evaluate strategies to improve availability and reach of these programs.

\section{MANUAL THERAPIES}

Though a variety of manual therapies are now well-established in the treatment of painful conditions, this workgroup identified several remaining challenges to studying manual therapies among individuals with chronic pain. These include the need to address heterogeneous pain; heterogeneity in delivery of manual therapies (e.g., variation in treatment techniques, dosing, and multimodal approaches); and identifying suitable sham comparators. Nevertheless, given the established effectiveness and favorable safety profiles, the workgroup cited a need for implementation research to enhance uptake of and accessibility to manipulation and acupuncture. Specifically, 
there is sufficient evidence to recommend dissemination of manipulation for low back pain (LBP) and neck pain; acupuncture for LBP, neck pain, mild to moderate knee osteoarthritis, and migraine and tension-type headache; and massage for LBP and neck pain.

The workgroup identified several other research priorities. Condition-based research priorities for manipulation should include determining specific effectiveness for radiculopathy, cervicogenic headache, thoracic spine pain, and extremity conditions; for acupuncture: effectiveness for advanced knee osteoarthritis, cervicogenic headache, and extremity conditions; and for massage: effectiveness for fibromyalgia, mild to moderate knee osteoarthritis, and tension-type headache. Across all manual therapies, there is a need for research on populations not typically well-represented in clinical trials to date such as older adults, as well as those with postoperative spine pain, severe osteoarthritis, current opioid use, and complex comorbidities. Several cross-cutting research priorities related to treatment planning were identified for all manual therapies; these are discussed below in the cross-cutting research section.

\section{MODELS FOR DELIVERING MULTI-MODAL PAIN CARE}

This workgroup identified two care model paradigms for which there is evidence for efficacy/effectiveness: collaborative care, where a care manager (e.g., nurse or pharmacist) teams with a physician to provide consultative, multi-modal pain care ${ }^{27,28}$, and stepped care, in which a care manager, following a defined algorithm, guides patients through a sequence of multi-modal treatments. ${ }^{29}$ However, the strength of evidence for these models is generally low. Thus, the workgroup recommended developing further evidence to address shortcomings of previous studies. Specifically, there is a need to better characterize patients' pain duration, opioid use, and prevalence of comorbidities and co-interventions received. There is also a need for more rigorous evaluations of model fidelity, assessment of a broader range of clinically relevant core outcomes, ${ }^{30}$ longer term follow-up, and inclusion of underserved populations such as rural Veterans and racial/ethnic minorities.

The workgroup observed that effective models are not reaching sufficient numbers of patients in part due to the lack of evidenced-based implementation strategies. Real-world dissemination challenges include the limited availability of care managers trained in pain management; the lack of cost effectiveness data; the fragmented nature of pain care services even in integrated systems; patient complexity complicating treatment decisions; lack of provider training in algorithm-guided pain treatment; and variability in patient education, activation, and expectations.

Novel models of care delivery need formal efficacy/ effectiveness studies, including peer-delivered or informal care-giver interventions, pharmacist-led pharmacological treatment optimization, group-delivered interventions, telecare-delivered interventions, and prognostic screening or risk stratification to tailor interventions. Other key issues for future effectiveness research included the following: how to optimize treatment effects in terms of matching resources to patient needs; the effect of tailoring interventions based on patient complexity; and exploring sequencing vs. combining intervention effects.

\section{CROSS-CUTTING RESEARCH ISSUES}

Across all work groups, common themes emerged about the need for further research involving tailoring therapies to patient preference, especially in light of roughly equivalent effect sizes across modalities; combining or sequencing therapies and/or treatment components; evaluating the optimal dose and modality for treatment delivery (e.g., telehealth vs. inperson sessions, individual vs. group, brief vs. standard length therapies); demographic and clinical characteristics predicting response to treatment; and optimizing duration of effect; as well as determining optimal approaches for those with comorbid mental health or complex medical conditions.

Yet, conducting standard randomized clinical trials to evaluate these various treatment features among patients with a range of clinical characteristics may not be feasible. Workgroups discussed innovative trial designs ${ }^{31}$ that allow rigorous, systematic ways of testing a combination of approaches within a single trial including the multiphase optimization strategy and the sequential multiple assignment randomized trial. ${ }^{32,33}$ These methods are designed to optimize intervention components with regard to dose and sequencing and to test which intervention components can be used alone or in combination. These types of study designs could also efficiently advance our relatively limited understanding of patient characteristics that predict response to different treatments.

Further, in the context of recognition of the weaknesses and limitations of pharmacologic trials and the failed clinical paradigms emanating from them, there have been increasing calls to consider a broader range of potentially important patientand system-level outcomes associated with pain treatments, including prioritizing improvements of pain-related functioning and quality of life (rather than a primary focus on reducing pain intensity), earlier and more systematic inclusion of adverse events, and consideration of costs and cost-effectiveness for individuals healthcare systems. Systematic consideration of such outcomes may highlight the relative benefits of the types of behavioral approaches described in this review.

\section{CONCLUSION}

There is widespread consensus that chronic pain management should feature non-pharmacologic treatments that foster patient self-management and biopsychosocial wellness. 
However, to bring this vision to reality, more evidenceestablishing research is needed. Based on the findings of a two-day VHA SOTA conference, we have outlined a research agenda related to four areas of focus. Within each area, we identified research gaps based on our assessment of the current state of the science that may be helpful in guiding future studies.

Acknowledgements: The authors wish to acknowledge the SOTA COchairs and planning committee members who were not involved in writing the manuscript but whose process and products are described herein: Robert D. Kerns, PhD; Erin E. Krebs, MD, MPH; Benjamin Kligler, MD, MPH; Jennifer Murphy, PhD; Stephen Ezeji-Okoye, MD; Daniel Cherkin, $\mathrm{PhD}$; Rollin "Mac" Gallagher, MD, MPH; and Matthew J. Bair, MD, MS.

Corresponding Author: William C. Becker, MD; Pain Research, Informatics, Multimorbidities \& Education Center of Innovation VA Connecticut Healthcare System, West Haven, CT 06516, USA (e-mail: william.becker@yale.edu).

Funders The Department of Veterans Affairs, Health Services Research and Development provided funds for the SOTA described in this manuscript. Drs. Becker and Heapy receive funding from VA HSR\&D/QUERI project 15-460. Dr. Makris was supported by the Center for Translational Medicine, NIH/NCATS Grants (KL2TROO1103 and UL1TR001105), and she is currently supported by a VA HSR\&D Career Development award (IK2HX001916). Dr. Krein is supported by a HSR\&D Research Career Scientist Award (RCS 11-222). Dr. Allen is supported by the Center for Health Services Research in Primary Care at the Durham VA Healthcare System (CIN 13-410) and a National Institute of Arthritis and Musculoskeletal and Skin Diseases Multidisciplinary Clinical Research Center P60 AR062760.

\section{Compliance with Ethical Standards:}

Conflict of Interest: Drs. Heapy and Higgins received consulting fees from Magellan Health for the development of a web-based CBT program for chronic pain. All other authors declare no conflicts of interest.

\section{REFERENCES}

1. Haskell SG, Ning Y, Krebs E, et al. Prevalence of painful musculoskeletal conditions in female and male veterans in 7 years after return from deployment in Operation Enduring Freedom/Operation Iraqi Freedom. Clin J Pain 2012;28(2): 163-167.

2. Chou R, Turner JA, Devine EB, et al. The effectiveness and risks of long-term opioid therapy for chronic pain: a systematic review for a National Institutes of Health Pathways to Prevention Workshop. Ann Intern Med 2015;162(4):276-286.

3. Dowell D, Haegerich T, Chou R. CDC Guideline for Prescribing Opioids for Chronic Pain - United States, 2016. MMWR Recomm Rep. 2016.

4. The Opioid Therapy for Chronic Pain Work Group. VA/DoD CLINICAL PRACTICE GUIDELINE FOR OPIOID THERAPY FOR CHRONIC PAIN. 2017; http://www.healthquality.va.gov/guidelines/Pain/cot/. Accessed May 16, 2017.

5. Hoffman BM, Papas RK, Chatkoff DK, Kerns RD. Meta-analysis of psychological interventions for chronic low back pain. Health Psychol: Offl J Div Health Psychol, Am Psychol Assoc 2007;26(1):1-9.

6. Dixon KE, Keefe FJ, Scipio CD, Perri LM, Abernethy AP. Psychological interventions for arthritis pain management in adults: a meta-analysis. Health Psychol: Offl J Div Health Psychol, Am Psychol Assoc 2007;26(3):241-250.

7. Glombiewski JA, Sawyer AT, Gutermann J, Koenig K, Rief w, Hofmann SG. Psychological treatments for fibromyalgia: a meta-analysis. Pain 2010;151(2):280-295.

8. Hughes LS, Clark J, Colclough JA, Dale E, McMillan D. Acceptance and Commitment Therapy (ACT) for Chronic Pain: A Systematic Review and Meta-Analyses. Clin J Pain 2017;33(6):552-568.
9. Veehof MM, Oskam MJ, Schreurs KM, Bohlmeijer ET. Acceptancebased interventions for the treatment of chronic pain: a systematic review and meta-analysis. Pain. 2011;152(3):533-542.

10. Cherkin DC, Sherman KJ, Balderson BH, et al. Effect of MindfulnessBased Stress Reduction vs Cognitive Behavioral Therapy or Usual Care on Back Pain and Functional Limitations in Adults With Chronic Low Back Pain: A Randomized Clinical Trial. JAMA. 2016;315(12): 1240-1249.

11. Becker WC, Dorflinger L, Edmond SN, Islam L, Heapy AA, Fraenkel L. Barriers and facilitators to use of non-pharmacological treatments in chronic pain. BMC Fam Pract 2017;18(1):41.

12. Macea DD, Gajos K, Daglia Calil YA, Fregni F. The efficacy of Webbased cognitive behavioral interventions for chronic pain: a systematic review and meta-analysis. J Pain 2010;11(10):917929.

13. Bender JL, Radhakrishnan A, Diorio C, Englesakis M, Jadad AR. Can pain be managed through the Internet? A systematic review of randomized controlled trials. Pain 2011;152(8):1740-1750.

14. Goulet JL, Kerns RD, Bair M, et al. The musculoskeletal diagnosis cohort: examining pain and pain care among veterans. Pain 2016;157(8): 1696-1703.

15. Hempel S, Taylor SL, Solloway MR, et al. Evidence map of tai chi. Department of Veterans Affairs: VA Evidence-based Synthesis Program Reports. 2014.

16. Wang $\mathbf{C}$, Schmid CH, Iversen MD, et al. Comparative Effectiveness of Tai Chi Versus Physical Therapy for Knee OsteoarthritisA Randomized TrialTai Chi Versus Physical Therapy for Knee Osteoarthritis. Ann Intern Med 2016;165(2):77-86.

17. Chou R, Deyo R, Friedly J, et al. Nonpharmacologic Therapies for Low Back Pain: A Systematic Review for an American College of Physicians Clinical Practice GuidelineNonpharmacologic Therapies for Low Back Pain. Ann Intern Med 2017;166(7):493-505.

18. Duan-Porter W, Coeytaux RR, McDuffie JR, et al. Evidence Map of Yoga for Depression, Anxiety, and Posttraumatic Stress Disorder. J Phys Act Health 2016;13(3):281-288.

19. Saper RB, Lemaster C, Delitto A, et al. Yoga, Physical Therapy, or Education for Chronic Low Back PainA Randomized Noninferiority TrialYoga, Physical Therapy, or Education for Chronic Low Back Pain. Ann Intern Med. 2017.

20. Moonaz SH, Bingham CO, Wissow L, Bartlett SJ. Yoga in sedentary adults with arthritis: effects of a randomized controlled pragmatic trial. J Rheumatol. 2015:jrheum. 141129.

21. Gaseem A, Wilt TJ, McLean RM, Forciea MA. Noninvasive Treatments for Acute, Subacute, and Chronic Low Back Pain: A Clinical Practice Guideline From the American College of PhysiciansNoninvasive Treatments for Acute, Subacute, and Chronic Low Back Pain. Ann Intern Med 2017;166(7):514-530.

22. Meng XG, Yue SW. Efficacy of aerobic exercise for treatment of chronic low back pain: a meta-analysis. Am J Phys Med Rehabil 2015;94(5):358-365.

23. O'Connor SR, Tully MA, Ryan B, et al. Walking exercise for chronic musculoskeletal pain: systematic review and meta-analysis. Arch Phys Med Rehabil 2015;96(4):724-734 e723.

24. Searle A, Spink M, Ho A, Chuter V. Exercise interventions for the treatment of chronic low back pain: a systematic review and meta-analysis of randomised controlled trials. Clin Rehabil 2015;29(12):1155-1167.

25. Wang SY, Olson-Kellogg B, Shamliyan TA, Choi JY, Ramakrishnan R, Kane RL. Physical therapy interventions for knee pain secondary to osteoarthritis: a systematic review. Ann Intern Med 2012;157(9):632-644.

26. Bartels EM, Lund H, Hagen $\mathbf{K B}$, Dagfinrud $\mathbf{H}$, Christensen $\mathbf{R}$, Danneskiold-Samsøe B. Aquatic exercise for the treatment of knee and hip osteoarthritis. Cochrane Database Syst Rev. 2007;4.

27. Dobscha SK, Corson K, Perrin NA, et al. Collaborative care for chronic pain in primary care: A cluster randomized trial. JAMA 2009;301(12): 1242-1252.

28. Kroenke K, Krebs EE, Wu J, Yu Z, Chumbler NR, Bair MJ. Telecare collaborative management of chronic pain in primary care: A randomized clinical trial. JAMA. 2014;312(3):240-248.

29. Bair MJ, Ang D, Wu J, et al. Evaluation of stepped care for chronic pain (escape) in veterans of the iraq and afghanistan conflicts: A randomized clinical trial. JAMA Intern Med 2015;175(5):682-689.

30. Dworkin RH, Turk DC, Farrar JT, et al. Core outcome measures for chronic pain clinical trials: IMMPACT recommendations. Pain 2005; 113(1-2):9-19.

31. Collins LM, Dziak JJ, Li R. Design of experiments with multiple independent variables: a resource management perspective on 
complete and reduced factorial designs. Psychol Methods 2009; 14(3):202-224.

32. Collins LM, Murphy SA, Strecher V. The multiphase optimization strategy (MOST) and the sequential multiple assignment randomized trial (SMART): new methods for more potent eHealth interventions. Am J Prev Med 2007;32(5 Suppl):S112-118.
33. Almirall D, Nahum-Shani I, Sherwood NE, Murphy SA. Introduction to SMART designs for the development of adaptive interventions: with application to weight loss research. Transl Behav Med 2014;4(3):260274. 